\title{
Large Data Considerations in Digital Holographic Microscopy
}

\author{
Carl Snyder ${ }^{*}$, David Cohoe ${ }^{1}$, Maximilian Schadegg ${ }^{1}$ and Jay Nadeau ${ }^{1}$ \\ 1. Department of Physics, Portland State University, Portland, OR, USA. \\ * Corresponding author: car15@pdx.edu
}

Digital holographic microscopy (DHM) is an interferometric technique with several advantages over traditional light microscopy. A hologram is an interference pattern produced by a coherent "reference" beam recombining with an "object" beam from the same source but that has passed through a sample (Fig. 1). The interference fringes encode the phase and amplitude of the object beam at the detector and can be used to calculate their values at any point along the path of the object beam (e.g. within the sample volume). These values can be used to calculate an intensity image (equivalent to brightfield) and a "phase" image that displays the index of refraction times the thickness of the objects of that index along the path. Resolution is the same as a conventional optical microscope with a comparable objective, but for typical optics needed to image prokaryotes, no loss in resolution is seen with samples as thick as a millimeter. This represents an approximately 100-fold improvement in depth of field over high-power light microscopy. No focusing is required and there are no moving parts. Plane-by-plane images are obtained through numerical reconstruction $[1,2]$; all z-plane information is encoded in a single hologram.

Reconstructed video stacks are extremely large. 30s of recording at $15 \mathrm{fps}$ at $2048 \times 2048$ results in a reconstructed data volume of over $0.5 \mathrm{~Tb}$. Methods for handling, visualizing, analyzing, presenting, and curating DHM data are in their infancy. In this work we present our laboratory's ongoing efforts to optimize DHM data analysis, minimize data volume for field and space applications, and to curate a public database of microbial motility videos.

One approach to DHM data analysis is to perform full reconstructions of all holograms, and adapt existing tools for electron and fluorescence microscopy that enable processing of large hyperstacks. Commercial solutions (arivis Vision4D) and open source solutions (Fiji [3]) have both been used with success in our laboratory, and workflows are shown here. We have created a set of Fiji plug-ins to reconstruct and process DHM data [4]. The resulting stacks may be customized for import into commercial software packages of choice, or into other Fiji plug-ins such as Big Data Viewer [5].

Another approach is to develop algorithms that can select image sequences likely to be scientifically valuable by analyzing the raw hologram without reconstructing image stacks. We have made progress in this area using machine learning and wavelet-based approaches [6, 7]. Our ongoing studies, presented here, focus on machine learning algorithms trained on data tracked in both holograms and image stacks are being used to select scientifically interesting image sequences. The training sets include mixed populations of inorganic particles and organisms of a range of sizes. While the training process is computationally intensive, the output is small set of parameters that could be used by efficient search algorithms.

When there are extreme limits on data volume, microbial motility data may be reduced to a series of tracks. Computation of power spectra reveals trends and periodicities in the frequency of swimming cycles, and enables direct visualization of spectral frequency differences between motile and non-motile cells in different environments. This may enable the identification of life in remote environments, such as the 
open ocean or the outer planets, with very little data volume requirement.

References:

[1] U Schnars and W Jueptner in "Digital holography : Digital Hologram Recording, Numerical Reconstruction, and Related Techniques", (Springer, Berlin).

[2] MK Kim in "Digital Holographic Microscopy : Principles, Techniques, and Applications", (Springer, New York).

[3] J Schindelin et al., Nature Methods 9 (2012), p. 676.

[4] D Cohoe et al., Frontiers in Physics in press (2019), p.

[5] T Pietzsch et al., Nat Methods 12 (2015), p. 481.

[6] M Bedrossian et al., AIMS Biophysics 5 (2018), p. 36.

[7] Z Marin et al., Methods 136 (2018), p. 60.
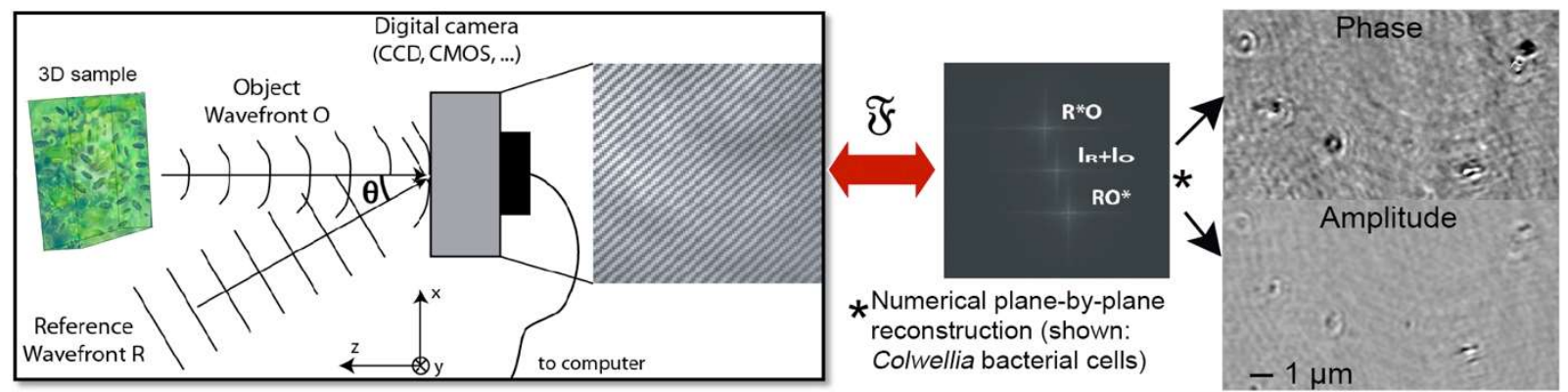

Figure 1. Off-axis DHM uses the interference of two coherent beams of light from a single source to record the complete sample volume in each captured frame. The "object" beam passes through the sample, while the "reference" beam takes an identical-length unperturbed path to the detector. The two beams are interfered at the detector array and the resulting interference pattern records the phase and amplitude of the object beam.

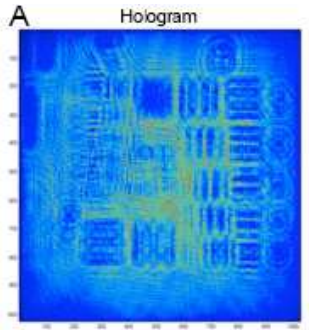

Record Hologram $\mathrm{H}$

$4 \mathrm{MB}$

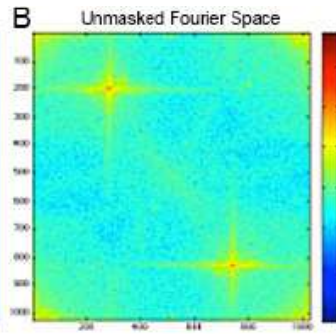

Fourier Transform

$4 \mathrm{MB}$

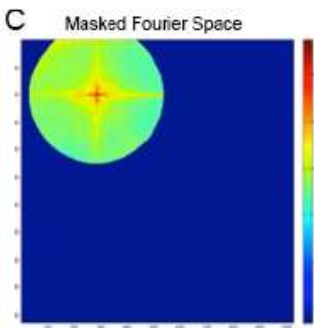

Mask to Leave Real Image

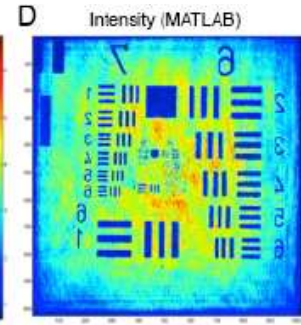

Digital focus construct image

$\sim 1$ GB $(125$ slices * 2$)$

Figure 2. Data types in DHM. A 4MB hologram becomes $\sim 1 \mathrm{~GB}$ when reconstructed into a full $\mathrm{z}$ stack in amplitude and phase. 